\title{
FRACTIONAL MOMENTS OF SOLUTIONS TO STOCHASTIC RECURRENCE EQUATIONS
}

\author{
THOMAS MIKOSCH, ${ }^{*}$ University of Copenhagen \\ GENNADY SAMORODNITSKY,** Cornell University \\ LALEH TAFAKORI, ${ }^{* * *}$ Shiraz University
}

\begin{abstract}
In this paper we study the fractional moments of the stationary solution to the stochastic recurrence equation $X_{t}=A_{t} X_{t-1}+B_{t}, t \in \mathbb{Z}$, where $\left(\left(A_{t}, B_{t}\right)\right)_{t \in \mathbb{Z}}$ is an independent and identically distributed bivariate sequence. We derive recursive formulae for the fractional moments $\mathbb{E}\left|X_{0}\right|^{p}, p \in \mathbb{R}$. Special attention is given to the case when $B_{t}$ has an Erlang distribution. We provide various approximations to the moments $\mathbb{E}\left|X_{0}\right|^{p}$ and show their performance in a small numerical study.
\end{abstract}

Keywords: Moment; stochastic recurrence equation; GARCH; Erlang distribution; numerical approximation

2010 Mathematics Subject Classification: Primary 60G70

Secondary 60K 99

\section{Introduction}

We consider the stochastic recurrence equation

$$
X_{t}=A_{t} X_{t-1}+B_{t}, \quad t \in \mathbb{Z},
$$

for an independent and identically distributed (i.i.d.) sequence $\left(\left(A_{t}, B_{t}\right)\right)_{t \in \mathbb{Z}}$ of pairs $\left(A_{t}, B_{t}\right)$ with values in $[0, \infty) \times \mathbb{R}$. We will write $A, B, C, \ldots$ for a generic variable of the stationary sequences $\left(A_{t}\right),\left(B_{t}\right),\left(C_{t}\right), \ldots$, respectively. A unique causal stationary solution to (1.1) exists if $\mathbb{E} \log A<0$ and $\mathbb{E} \log B^{+}<\infty$ (see [25]), and the solution can be written in the form

$$
X_{t}=B_{t}+\sum_{i=-\infty}^{t-1} A_{i+1} \cdots A_{t} B_{i}, \quad t \in \mathbb{Z} .
$$

In what follows, we always assume that the stationary solution (1.2) exists.

Received 15 March 2012; revision received 6 March 2013.

* Postal address: Department of Mathematics, University of Copenhagen, Universitetsparken 5, DK-2100 Copenhagen, Denmark. Email address: mikosch@math.ku.dk

Research partly supported by the Danish Natural Science Research Council (FNU) grant 10-084172 'Heavy tail phenomena: Modeling and estimation'.

** Postal address: School of Operations Research and Industrial Engineering, Cornell University, 220 Rhodes Hall, Ithaca, NY 14853, USA. Email address: gennady@orie.cornell.edu

Research partially supported by the ARO grant W911NF-07-1-0078, NSF grant DMS-1005903, and NSA grant H9823011-1-0154 at Cornell University.

*** Postal address: Department of Statistics, Shiraz University, College of Sciences, Shiraz, 7146713565, Iran. Email address: tafakori@ shirazu.ac.ir

This paper was written when Laleh Tafakori visited the Department of Mathematics at the University of Copenhagen in 2011. She takes pleasure to thank the host institution for its hospitality. 
The stochastic recurrence equation (1.1) and its solution (1.2) have attracted significant attention in the literature; see, e.g. [12], [21], [25], [33], and the references therein. This interest is due to the numerous applications of the model (1.1). Among the most popular applications are the $\mathrm{ARCH}(1)$ and $\operatorname{GARCH}(1,1)$ processes in financial time series analysis introduced by Engle [18] and Bollerslev [6], respectively. Another recent application is the modeling of the TCP in telecommunications networks; see, e.g. [17], [23], and [28]. Boxma et al. [7] considered (1.1) in the context of growth-collapse processes with renewal collapse epochs. The stochastic recurrence equation (1.1) has also been used in the context of insurance risk models; see [11] and [20]. Moreover, this equation is closely related to exponential functionals of Lévy processes; see, e.g. [5], [9], [10], [24], and [29].

The distributional properties of the stationary solution to (1.1) are rather sophisticated. This fact is highlighted by a famous result of Kesten [25] concerning the tails of $X$; see also [21]. Write

$$
f(\kappa)=\mathbb{E} A^{\kappa} \quad \text { and } \quad \phi(\kappa)=1-f(\kappa), \quad \kappa \in \mathbb{R} .
$$

Under the assumptions that there exists a positive $\alpha$ such that $f(\alpha)=1, \mathbb{E} A^{\alpha} \log A$ and $\mathbb{E}|B|^{\alpha}$ are both finite, the law of $\log A$ is nonarithmetic, and, for every $x, \mathbb{P}\left(A_{1} x+B_{1}=x\right)<1$, there exist constants $c_{+}, c_{-} \geq 0$ such that $c_{+}+c_{-}>0$, and

$$
\mathbb{P}(X>x) \sim c_{+} x^{-\alpha} \text { and } \mathbb{P}(X \leq-x) \sim c_{-} x^{-\alpha} \quad \text { as } x \rightarrow \infty .
$$

Because of the convexity of $f$ and since $f(0)=1$, we necessarily have $f(\kappa)<1$ for $\kappa \in(0, \alpha)$. Goldie [21] gave an alternative proof of (1.3) and determined the explicit form of the constants $c_{+}$and $c_{-}$. In particular, for $A, B \geq 0$, he proved that

$$
c_{+}=\frac{\mathbb{E}\left[\left(A_{1} X_{0}+B_{1}\right)^{\alpha}-\left(A_{1} X_{0}\right)^{\alpha}\right]}{\alpha \mathbb{E} A^{\alpha} \log A} .
$$

Note that, due to the tail behavior (1.3), $\mathbb{E} X^{\alpha}=\infty$; hence, both $\mathbb{E}\left(A_{1} X_{0}+B_{1}\right)^{\alpha}$ and $\mathbb{E}\left(A_{1} X_{0}\right)^{\alpha}$ are infinite while the nominator in the previous formula is finite.

If $A \leq 1$ almost surely (a.s.), $\mathbb{P}(0<A<1)>0$, and $\mathbb{E} \mathrm{e}^{r|B|}<\infty$ for some $r>0$, Goldie and Grübel [22] showed that (1.3) does not remain true. In this case, the tails of $X$ decay exponentially fast, thus ensuring the existence of all moments of $X$. If we formally set $\alpha=\infty$, we have $f(\kappa)=\mathbb{E} A^{\kappa}<1$ for $\kappa<\alpha$, just as in the Kesten-Goldie case. Under mild conditions on $A$ and $B$, Alsmeyer et al. [1] showed that $A \leq 1$ a.s. and the existence of exponential moments of $B$ are conditions which are necessary to ensure that exponential moments of $X$ exist. In the same paper, the authors quote a technical report of Kellerer (1992), who proved for $A, B \geq 0$ a.s. that $\mathbb{E}^{r X}<\infty$ for some $r>0$ if and only if $A \leq 1$ a.s. and $\mathbb{E} \mathrm{e}^{r B}<\infty$.

In this paper we are concerned with the calculation of the moments of the solution $\left(X_{t}\right)_{t \in \mathbb{Z}}$ to the stochastic recurrence equation (1.1). The positive integer moments of $X$ can be calculated by using the recursive argument given in [33]. First, observe that, from (1.1) for integer $n \geq 1$,

$$
X^{n} \stackrel{\mathrm{D}}{=}\left(A_{1} X_{0}+B_{1}\right)^{n},
$$

where $X_{0}$ and $\left(A_{1}, B_{1}\right)$ are independent. Then, assuming that $\mathbb{E}|X|^{p}$ is finite and $f(p)<1$ for some $p \geq 1$, an application of the binomial formula yields a recursive relation for the moments $\mathbb{E} X^{l}, 1 \leq l \leq n=[p]$, given by

$$
\mathbb{E} X^{l}=(\phi(l))^{-1} \sum_{k=0}^{l-1}\left(\begin{array}{l}
l \\
k
\end{array}\right) \mathbb{E}\left(A_{1}^{k} B_{1}^{l-k}\right) \mathbb{E} X^{k} .
$$


Note that $f(l)<1, l \leq n$, if $p<\alpha$ for the value $\alpha \leq \infty$ introduced above. It follows from [1, Theorem 1.4] that $\mathbb{E}|X|^{p}<\infty$ if and only if $\mathbb{E} A^{p}<1$ and $\mathbb{E}|B|^{p}<\infty$ for any $p>0$, provided that the mild conditions $A \neq 0$ a.s., $\mathbb{P}(B=0)<1$, and $\mathbb{P}\left(A_{1} x+B_{1}=x\right)<1, x \in \mathbb{R}$, hold.

In some special cases the distribution of $X$ can be calculated explicitly; see, e.g. [1], [7], [13], [14], [15], [16], [21], [30], and the references therein. Then, in principle, one could also calculate all moments of $X$, both the integer and the fractional moments.

In this paper we focus on the derivation of explicit formulae for the fractional moments of $X$. Such moments were derived for models of telecommunication networks in [10] and [23], and for exponential functionals of Lévy processes in [10], [23], and [29]. We also mention that, for exponential $B$, the calculation of Goldie's constant $c_{+}$in (1.4) reduces to calculating the fractional moment $\mathbb{E} X^{\alpha-1}$; see the comments at the end of Example 2.1. Moments are highly relevant in statistical and econometric applications. In financial time series analysis, the ARCH and GARCH processes introduced by Engle [18] and Bollerslev [6], respectively, constitute a major class which is closely related to multivariate stochastic recurrence equations of the type (1.1); see, e.g. [3] and [4]. The $\operatorname{GARCH}(1,1)$ process fits into the one-dimensional stochastic recurrence equation framework: it is a stationary process $Y_{t}=\sigma_{t} Z_{t}, t \in \mathbb{Z}$, where $\left(Z_{t}\right)$ is a mean zero, unit variance i.i.d. sequence and $\left(\sigma_{t}^{2}\right)$ solves the stochastic recurrence equation

$$
\sigma_{t}^{2}=\alpha_{0}+\left(\alpha_{1} Z_{t-1}^{2}+\beta_{1}\right) \sigma_{t-1}^{2}, \quad t \in \mathbb{Z},
$$

for positive coefficients $\alpha_{i}$ and $\beta_{1}$. In applications $Z$ is often assumed standard normal or student distributed with $\beta>2$ degrees of freedom. Then one is in the Kesten-Goldie framework, i.e. there exist positive $c_{0}, \alpha>0$ with $\mathbb{E}\left(\alpha_{1} Z^{2}+\beta_{1}\right)^{\alpha}=1$ such that $\mathbb{P}\left(\sigma^{2}>x\right) \sim c_{0} x^{-\alpha}$, and a result of Breiman [8] ensures that the distribution of $Y$ inherits the power law tail of $\sigma$. In applications we are interested in the moments $\mathbb{E}|Y|^{p}=\mathbb{E} \sigma^{p} \mathbb{E}|Z|^{p}, p>0$, as well as in the correlations of the sequences $\left(\left|Y_{t}\right|^{p}\right)$ for positive $p$, most often for $p=1,2$.

The paper is organized as follows. In Section 2 we consider some recursive formulae for the moments of $X$. In Section 3 we consider some special cases. Our main focus is on cases which are related to exponential random variables $B_{t}$. We use the results and techniques proved in [10] and [23]. We also consider the case when $0 \leq A<1$ and $B$ is bounded, and derive explicit formulae for $\mathbb{E} X^{p}$.

\section{Preliminaries}

\subsection{A simple recursive formula for moments}

For the calculation of the fractional moments, the following observation is useful. A similar formula was applied for calculating the moments of exponential functionals of Lévy processes in [10], [23], and [29]. In what follows, we write $F_{Z}$ for the distribution function of any random variable $Z$ and $\bar{F}_{Z}=1-F_{Z}$ for its right tail.

Lemma 2.1. Let $p \neq 0$ be any real number. Assume that $A, B \geq 0$ a.s. are independent. Then

$$
\mathbb{E}\left[\left(A_{1} X_{0}+B_{1}\right)^{p}-\left(A_{1} X_{0}\right)^{p}\right]=p \int_{0}^{\infty} \mathbb{E}\left(A_{1} X_{0}+u\right)^{p-1} \bar{F}_{B}(u) \mathrm{d} u,
$$

where both sides are finite or infinite at the same time. If $0<\mathbb{E} B<\infty$, (2.1) can be written in the form

$$
\mathbb{E}\left[\left(A_{1} X_{0}+B_{1}\right)^{p}-\left(A_{1} X_{0}\right)^{p}\right]=p \mathbb{E} B \mathbb{E}\left(A_{1} X_{0}+B^{*}\right)^{p-1}
$$


for a random variable $B^{*}$ which is independent of $A_{1}, X_{0}$ and has the integrated tail distribution of $B$ given by

$$
F_{B}^{*}(b)=\frac{\int_{0}^{b} \bar{F}_{B}(u) \mathrm{d} u}{\mathbb{E} B}, \quad b>0 .
$$

Proof. We observe that, for any $p \in \mathbb{R}$,

$$
\left(A_{1} X_{0}+B_{1}\right)^{p}-\left(A_{1} X_{0}\right)^{p}=p \int_{0}^{B_{1}}\left(A_{1} X_{0}+u\right)^{p-1} \mathrm{~d} u .
$$

Hence, by the independence of $A_{1} X_{0}$ and $B_{1}$,

$$
\begin{aligned}
\mathbb{E}\left[\left(A_{1} X_{0}+B_{1}\right)^{p}-\left(A_{1} X_{0}\right)^{p}\right] & =p \mathbb{E}\left[\int_{0}^{B_{1}}\left(A_{1} X_{0}+u\right)^{p-1} \mathrm{~d} u\right] \\
& =p \int_{0}^{\infty}\left[\int_{0}^{b} \mathbb{E}\left(A_{1} X_{0}+u\right)^{p-1} \mathrm{~d} u\right] F_{B}(\mathrm{~d} b) \\
& =p \int_{0}^{\infty} \mathbb{E}\left(A_{1} X_{0}+u\right)^{p-1} \bar{F}_{B}(u) \mathrm{d} u .
\end{aligned}
$$

Then the statement of the lemma follows.

Remark 2.1. If $\mathbb{E} X^{p}<\infty, \mathbb{E} B<\infty$, and $f(p) \neq 1$, then the lemma yields

$$
\mathbb{E} X^{p}=\frac{p \mathbb{E} B}{\phi(p)} \mathbb{E}\left(A_{1} X_{0}+B^{*}\right)^{p-1} .
$$

Following the argument after (1.5), we conclude that $f(p)<1$ is a necessary condition for $\mathbb{E} X^{p}<\infty$ provided $p>0$ and some mild conditions on $A, B$ are satisfied. Since $f$ is convex and $f(0)=1, f(p)>1$ for $p<0$. Hence, $f(p) \neq 1$ is satisfied for all $p \neq 0$ such that $f(p)$ is finite.

The idea of the proof of Lemma 2.1 can be applied iteratively. We explain the approach via an example. Assume the conditions of the lemma are satisfied. Write $F_{B}^{n *}$ for the distribution function of a random variable $B^{n *}$, which is obtained by applying the integrated tail operation (2.2) $n$ times and assuming that $B^{n *}$ is independent of $\left(A_{1}, X_{0}\right)$. Then, assuming that all moments involved are finite and $p \neq 0$,

$$
\begin{aligned}
\mathbb{E} X^{p} & =\frac{p \mathbb{E} B}{\phi(p)}\left[\mathbb{E}\left(A_{1} X_{0}+B^{*}\right)^{p-1}-\mathbb{E}\left(A_{1} X_{0}\right)^{p-1}+\mathbb{E}\left(A_{1} X_{0}\right)^{p-1}\right] \\
& =\frac{p \mathbb{E} B}{\phi(p)}\left[(p-1) \mathbb{E} B^{*} \mathbb{E}\left(A_{1} X_{0}+B^{2 *}\right)^{p-2}+\mathbb{E}\left(A_{1} X_{0}\right)^{p-1}\right] \\
& =\frac{p(p-1) \mathbb{E} B}{\phi(p)}\left[\mathbb{E} B^{*} \mathbb{E}\left(A_{1} X_{0}+B^{2 *}\right)^{p-2}+\frac{f(p-1) \mathbb{E} B}{\phi(p-1)} \mathbb{E}\left(A_{1} X_{0}+B^{*}\right)^{p-2}\right] .
\end{aligned}
$$

To illustrate the use of Lemma 2.1, we include the following benchmark example.

Example 2.1. Assume that $B$ has a standard exponential distribution, i.e. $\bar{F}_{B}(x)=\mathrm{e}^{-x}, x>0$. Then $B^{*} \stackrel{\mathrm{D}}{=} B$ and $A_{1} X_{0}+B^{*} \stackrel{\mathrm{D}}{=} X$. Multiple use of (2.3) yields

$$
\mathbb{E} X^{p}=\frac{p \cdots(p-n+1)}{\phi(p) \cdots \phi(p-n+1)} \mathbb{E} X^{p-n}, \quad n \geq 1 .
$$


Relation (2.4) can be found in the literature on exponential functionals of Lévy processes and related topics; see, e.g. [5], [9], [10], [24], and [29]. An explicit solution to (2.4) for $A_{t}=\beta^{Y_{t}}$, positive $Y_{t}, \beta \in(0,1)$, was given in [23]:

$$
\mathbb{E} X^{p}=\Gamma(p+1) \prod_{k=1}^{\infty} \frac{\phi(p+k)}{\phi(k)},
$$

provided $p>0$ or $p<0,-p \notin \mathbb{N}, f(p+1)<\infty$, and $\mathbb{E}\left[(1-A)^{-1}\right]<\infty$.

Under the assumptions of the Kesten-Goldie theory [21], [25] (see Section 1), the constant $c_{+}$in (1.4) satisfies

$$
\begin{aligned}
c_{+} \mathbb{E} A^{\alpha} \log A & =\alpha^{-1} \mathbb{E}\left[\left(A_{1} X_{0}+B_{1}\right)^{\alpha}-\left(A_{1} X_{0}\right)^{\alpha}\right] \\
& =\mathbb{E}\left(A_{1} X_{0}+B_{1}\right)^{\alpha-1} \\
& =\mathbb{E} X^{\alpha-1} \\
& <\infty
\end{aligned}
$$

although $\mathbb{E} X^{\alpha}=\infty$. Then, if $c_{+}$and $\mathbb{E} A^{\alpha} \log A$ are known, we can calculate the moments $\mathbb{E} X^{\alpha-k}, k=1,2, \ldots$, using (2.4). Alternatively, if we know $\mathbb{E} X^{\alpha-1}$ and $\mathbb{E} A^{\alpha} \log A$, we could calculate $c_{+}$. Unfortunately, (2.5) is not available in the Kesten-Goldie setting.

\subsection{A result about the convergence of moments}

The following result will be useful.

Lemma 2.2. Consider three i.i.d. sequences $\left(A_{t}\right),\left(B_{t}\right)$, and $\left(B_{t}^{(n)}\right)$ of nonnegative random variables defined on the same probability space. Assume that $\left(A_{t}\right)$ and $\left(B_{t}\right)$ are independent, and that $\left(A_{t}\right)$ and $\left(B_{t}^{(n)}\right)$ are also independent. Let $\left(X_{t}\right)$ be the solution to $(1.1)$, and let $\left(X_{t}^{(n)}\right)$ be the corresponding solution with $\left(B_{t}\right)$ replaced by $\left(B_{t}^{(n)}\right)$. If $\mathbb{E} A^{p}<1$ and $\mathbb{E}\left|B_{0}-B_{0}^{(n)}\right|^{p} \rightarrow 0$ as $n \rightarrow \infty$ for some $p>0$, then

$$
X^{(n)} \stackrel{\mathrm{D}}{\rightarrow} X \quad \text { and } \quad \mathbb{E}\left|X^{(n)}\right|^{p} \rightarrow \mathbb{E}|X|^{p} \quad \text { as } n \rightarrow \infty
$$

Proof. Recall the Mallows metric $d_{p}(R, S)=\inf \left(\mathbb{E}|R-S|^{p}\right)^{\min (1,1 / p)}, p>0$, where the infimum is taken over all joint distributions of the bivariate vectors $(R, S)$ with fixed marginals and $p$ th finite moments. It is well known (see, e.g. [31]) that $d_{p}$ metrizes convergence in distribution and $L^{p}$ convergence. Under the assumptions of the lemma, for $p \leq 1$,

$$
\begin{aligned}
d_{p}\left(X_{0}^{(n)}, X_{0}\right) & \leq \mathbb{E}\left|X_{0}^{(n)}-X_{0}\right|^{p} \\
& =\mathbb{E}\left|\sum_{i=-\infty}^{t} A_{i+1} \cdots A_{t}\left[B_{i}^{(n)}-B_{i}\right]\right|^{p} \\
& \leq \sum_{i=-\infty}^{t}\left(\mathbb{E} A^{p}\right)^{t-i} \mathbb{E}\left|B^{(n)}-B\right|^{p} .
\end{aligned}
$$

But $\sum_{i=-\infty}^{t}\left(\mathbb{E} A^{p}\right)^{t-i}<\infty$ since $\mathbb{E} A^{p}<1$ and $\mathbb{E}\left|B_{0}^{(n)}-B_{0}\right|^{p} \rightarrow 0$. Then (3.6) follows for $p \leq 1$. For $p>1$, the same idea of proof applies if we use the Minkowski inequality of order $p$ in (2.6).

The following example illustrates the use of Lemma 2.2. 
Example 2.2. Assume $\left(A_{t}\right)$ and $\left(B_{t}\right)$ are independent i.i.d. sequences of nonnegative random variables, and that $\left(X_{t}\right)$ solves (1.1). We also assume that $\mathbb{E} A^{p}<1$ and $\mathbb{E} B^{p}<\infty$ for some $p>0$. Let $B^{(a)}$ be a random variable with distribution given by

$$
\begin{aligned}
& \quad \mathbb{P}\left(B^{(a)}=0\right)=1-a \text { for some } a \in(0,1] \\
& \text { and } \quad \mathbb{P}\left(B^{(a)}>x\right)=a(1-\mathbb{P}(B \leq x)), \quad x>0 .
\end{aligned}
$$

Write $F_{a}$ for the distribution of $B^{(a)}$. Denote by $\left(X_{t}^{(a)}\right)$ the solution to (1.1) for independent i.i.d. sequences $\left(A_{t}\right)$ and $\left(B_{t}^{(a)}\right)$, where $B_{t}^{(a)} \stackrel{D}{=} B^{(a)}$. Consider an i.i.d. uniform sequence $\left(U_{i}\right)$ on $(0,1)$ independent of $\left(A_{t}\right)$. Then $F_{a}^{\leftarrow}\left(U_{t}\right)$ has the same distribution as $B^{(a)}$, where $F_{a}^{\leftarrow}$ denotes the quantile function of $F_{a}$. Obviously, $\mathbb{E}\left|B_{0}^{(a)}-B_{0}^{(1)}\right|^{p} \rightarrow 0$ as $a \rightarrow 1$. An application of Lemma 2.2 now yields

$$
X_{0}^{(a)} \stackrel{\mathrm{D}}{\rightarrow} X \quad \text { and } \quad \mathbb{E}\left|X_{0}^{(a)}\right|^{p} \stackrel{\mathrm{D}}{\rightarrow} \mathbb{E}|X|^{p}
$$

\section{Special cases}

In this section we consider several special choices for the distributions of $A$ and $B$, and give recursive or explicit expressions for the fractional moments of $X$.

\subsection{Cases related to exponential $B$}

The examples of this section are closely related to Example 2.1, i.e. to the case of exponentially distributed $B$. We will show how Lemma 2.1 and the results of Example 2.1 can be applied to classes of distributions of $B$ beyond the exponential distribution.

3.1.1. Erlang distributed B. In Example 2.1 we explained how Lemma 2.1 can be applied to exponentially distributed $B$. A natural extension is to assume that $B$ has an Erlang distribution.

Lemma 3.1. Assume that $\left(E_{i}\right)$ is an i.i.d. standard exponential, independent of $\left(A_{i}\right)$, and that $B \stackrel{\mathrm{D}}{=} \Gamma_{n}=E_{1}+\cdots+E_{n}$ for some $n \geq 1$. Also, assume that $A \geq 0$ a.s. and $A, B$ are independent. Then, for $p \in \mathbb{R}$,

$$
\phi(p) \mathbb{E} X^{p}=\sum_{k=1}^{n}(-1)^{k+1}\left(\begin{array}{l}
n \\
k
\end{array}\right) \mathbb{E} X^{p-k} p(p-1) \cdots(p-k+1),
$$

where we assume that all moments in this formula are finite.

Proof. For $n=1$, this is just (2.4). Now assume that $n \geq 2$. We have, with $\Gamma_{0}=0$,

$$
\begin{aligned}
\phi(p) \mathbb{E} X^{p} & =\mathbb{E}\left(A_{1} X_{0}+\Gamma_{n}\right)^{p}-\mathbb{E}\left(A_{1} X_{0}\right)^{p} \\
& =\sum_{l=1}^{n}\left[\mathbb{E}\left(A_{1} X_{0}+\Gamma_{l}\right)^{p}-\mathbb{E}\left(A_{1} X_{0}+\Gamma_{l-1}\right)^{p}\right] .
\end{aligned}
$$

As in the proof of Lemma 2.1, we obtain

$$
\mathbb{E}\left(A_{1} X_{0}+\Gamma_{l}\right)^{p}-\mathbb{E}\left(A_{1} X_{0}+\Gamma_{l-1}\right)^{p}=p \mathbb{E}\left(A_{1} X_{0}+\Gamma_{l}\right)^{p-1} .
$$

For $l=n, A_{1} X_{0}+\Gamma_{n} \stackrel{\mathrm{D}}{=} A_{1} X_{0}+B_{1} \stackrel{\mathrm{D}}{=} X$. Therefore,

$$
\mathbb{E}\left(A_{1} X_{0}+\Gamma_{n-1}\right)^{p}=\mathbb{E} X^{p}-p \mathbb{E} X^{p-1} .
$$


Using (3.2) and (3.3), we obtain

$$
\begin{aligned}
\mathbb{E}\left(A X+\Gamma_{n-1}\right)^{p}-\mathbb{E}\left(A X+\Gamma_{n-2}\right)^{p} & =p \mathbb{E}\left(A X+\Gamma_{n-1}\right)^{p-1} \\
& =p \mathbb{E} X^{p-1}-p(p-1) \mathbb{E} X^{p-2} .
\end{aligned}
$$

Induction yields, for $l=1, \ldots, n$,

$$
\begin{aligned}
\mathbb{E}\left(A_{1} X_{0}+\Gamma_{l}\right)^{p}-\mathbb{E}\left(A_{1} X_{0}+\Gamma_{l-1}\right)^{p} \\
=\sum_{j=0}^{n-l}(-1)^{j}\left(\begin{array}{c}
n-l \\
j
\end{array}\right) p(p-1) \cdots(p-j) \mathbb{E} X^{p-j-1} .
\end{aligned}
$$

This concludes the proof of (3.1).

Relation (3.1) yields a recursive relation for $\mathbb{E} X^{p}$ in terms of the lower moments $\mathbb{E} X^{p-1}, \ldots$, $\mathbb{E} X^{p-n}$. In contrast to the case $n=1$ (exponential $B$ ), we could not solve the recursion (3.1) explicitly. There is evidence that (3.1) yields good approximations to higher moments if one can approximate $\mathbb{E} X^{p}$ for small initial values of $p$ (e.g. by Monte Carlo simulation); this has already been reported in [12].

In the Kesten-Goldie setting, the right-hand side of (3.1) remains valid for the tail index $p=$ $\alpha$ if the left-hand side is replaced by $\mathbb{E}\left[\left(A_{1} X_{0}+\Gamma_{n}\right)^{\alpha}-\left(A_{1} X_{0}\right)^{\alpha}\right]$. The resulting formula yields an expression for Goldie's constant $c_{+}$in (1.4) in terms of the moments $\mathbb{E} X^{\alpha-1}, \ldots, \mathbb{E} X^{\alpha-n}$.

3.1.2. The distribution of $B$ is a multiplicative mixture of an exponential distribution. A particular case of (1.1) has attracted some attention:

$$
X_{t}=A_{t}\left(X_{t-1}+C_{t}\right), \quad t \in \mathbb{R},
$$

for independent sequences $\left(A_{t}\right)$ and $\left(C_{t}\right)$ of i.i.d. nonnegative random variables. In this case, $B_{t}=A_{t} C_{t}$, and $A_{t}$ and $B_{t}$ are dependent for every $t$ with the exception of constant $A$. The marginal distribution of the solution to (3.4) is known in some particular cases when $A$ and $C$ have gamma- or beta-like distributions; see [13], [14], [15], [16], and [7], [10].

Lemma 3.2. Consider model (3.4), and assume that $C$ is standard exponential and $\left(A_{t}\right)=$ $\left(\beta^{Y_{t}}\right)$ for some $\beta \in(0,1)$ and an i.i.d. sequence $\left(Y_{t}\right)$ with $Y>0$ a.s. Then

$$
\mathbb{E} X^{p}=f(p) \mathbb{E}\left(X_{0}+C_{1}\right)^{p}=f(p) \Gamma(p+1) \prod_{k=1}^{\infty} \frac{\phi(p+k)}{\phi(k)},
$$

provided $p>0$ or $p<0,-p \notin \mathbb{N}, f(p+1)<\infty$, and $\mathbb{E}(1-A)^{-1}<\infty$, and both sides in (3.5) are finite or infinite at the same time.

Proof. Assume that $\mathbb{E} X^{p}<\infty$ for some $p>0$. Then, by Lemma 2.1,

$$
\begin{aligned}
\mathbb{E} X^{p} & =f(p) \mathbb{E}\left(X_{0}+C_{1}\right)^{p} \\
& =f(p)\left[\mathbb{E}\left(X_{0}+C_{1}\right)^{p}-\mathbb{E} X^{p}+\mathbb{E} X^{p}\right] \\
& =f(p)\left[p \mathbb{E}\left(X_{0}+C_{1}\right)^{p-1}+\mathbb{E} X^{p}\right] .
\end{aligned}
$$

Hence,

$$
\mathbb{E}\left(X_{0}+C_{1}\right)^{p}=\frac{p}{\phi(p)} \mathbb{E}\left(X_{0}+C_{1}\right)^{p-1}
$$


Then one is in the situation of Example 2.1. Replacing in (2.4) the moments $\mathbb{E} X^{p-k}, k=$ $0,1, \ldots$, by the corresponding moments of $X_{0}+C_{1}$, the proof of Proposition 7 of [23] applies to conclude that

$$
\mathbb{E}\left(X_{0}+C_{1}\right)^{p}=\Gamma(p+1) \prod_{k=1}^{\infty} \frac{\phi(p+k)}{\phi(k)} .
$$

3.1.3. The distribution of $B$ is exponential with an atom at 0 . In this subsection we assume that

$$
\mathbb{P}(B=0)=1-a \quad \text { for some } a \in(0,1) \quad \text { and } \quad \mathbb{P}(B>x)=a \mathrm{e}^{-x} \quad \text { for } x>0 .
$$

Then $B^{*}$ has a standard exponential distribution and it is plausible that the results for exponential $B$ are applicable. This is the content of the next result.

Lemma 3.3. Assume that $B$ has the distribution (3.6), that $\left(A_{t}\right)=\left(\beta^{Y_{t}}\right)$ for some $\beta \in(0,1)$ and an i.i.d. sequence $\left(Y_{t}\right)$ of positive random variables, and that $\left(A_{t}\right)$ and $\left(B_{t}\right)$ are independent. Then, for $p>0$, the relation

$$
\mathbb{E} X^{p}=\Gamma(p+1) \prod_{k=1}^{\infty} \frac{\phi(p+k)}{\phi(k)} \frac{1-(1-a) \mathbb{E} A^{k-1}}{1-(1-a) \mathbb{E} A^{p+k-1}}
$$

holds, provided $\mathbb{E}\left[(1-A)^{-1}\right]<\infty$ and

$$
(1-a) \mathbb{E} A^{p-1}<1 \text { for } p \in(0,1)
$$

Proof. An application of Lemma 2.1 and (2.3) yields, for real $p$ and standard exponential $B$,

$$
\mathbb{E} X^{p}=\frac{p a}{\phi(p)} \mathbb{E}\left(A_{1} X_{0}+B^{*}\right)^{p-1}
$$

where both sides are finite or infinite at the same time. The moment on the right-hand side is finite for $p>0$. For $p \geq 1$, this is elementary, and, for $p \in(0,1)$, we have

$$
\mathbb{E}\left(A_{1} X_{0}+B^{*}\right)^{p-1} \leq \mathbb{E}\left(B^{*}\right)^{p-1}=\int_{0}^{\infty} x^{-(1-p)} \mathrm{e}^{-x} \mathrm{~d} x<\infty
$$

Under the assumption (3.8), we also have $\mathbb{E} X^{p-1}<\infty$ for $p \in(0,1)$, as we will show next. We have the representation $B_{i}=E_{i} r_{i}, i \in \mathbb{Z}$, where $\left(E_{i}\right)$ is an i.i.d. standard exponential sequence independent of the i.i.d. sequence $\left(r_{i}\right)$, where $\mathbb{P}\left(r_{i}=1\right)=1-\mathbb{P}\left(r_{i}=0\right)=a$. Now consider $T_{0}=0$ and $T_{1}=\min \left\{i \geq 1: r_{i}=1\right\}$. This random variable is geometrically distributed with success probability $a: \mathbb{P}\left(T_{1}=k\right)=a(1-a)^{k-1}, k \geq 1$. By induction, define $T_{i+1}=\min \left\{k>T_{i}: r_{k}=1\right\}, i \geq 1$. The sequence $\left(T_{i+1}-T_{i}\right)$ is i.i.d. Using the representation $X \stackrel{\mathrm{D}}{=} \sum_{i=0}^{\infty} B_{i+1} \Pi_{i}$, where $\Pi_{i}=\prod_{k=1}^{i} A_{k}$ with the convention that $\Pi_{0}=1$, 
we also have $X \stackrel{\mathrm{D}}{=} \sum_{k=1}^{\infty} E_{T_{k}} \Pi_{T_{k}-1}$. Hence,

$$
\begin{aligned}
\mathbb{E} X^{p-1} & \leq \mathbb{E}\left(E_{T_{1}} \Pi_{T_{1}}\right)^{p-1} \\
& =\sum_{k=1}^{\infty} a(1-a)^{k-1} \mathbb{E}\left(E_{k} \Pi_{k}\right)^{p-1} \\
& =\sum_{k=1}^{\infty} a(1-a)^{k-1} \mathbb{E}\left(B^{*}\right)^{p-1}\left(\mathbb{E} A^{p-1}\right)^{k} \\
& =a \mathbb{E} A^{p-1} \mathbb{E}\left(B^{*}\right)^{p-1}\left(1-(1-a) \mathbb{E} A^{p-1}\right)^{-1} \\
& <\infty,
\end{aligned}
$$

where we used (3.8) in the last step. Relation (3.9) can be written in the form

$$
\begin{aligned}
\mathbb{E} X^{p} & =\frac{p}{\phi(p)}\left[a \mathbb{E}\left(A_{1} X_{0}+B^{*}\right)^{p-1}+(1-a) \mathbb{E}\left(A_{1} X_{0}\right)^{p-1}\right]-(1-a) \mathbb{E}\left(A_{1} X_{0}\right)^{p-1} \\
& =\frac{p}{\phi(p)}\left[1-(1-a) \mathbb{E} A^{p-1}\right] \mathbb{E} X^{p-1} \\
& =\frac{p}{g(p)} \mathbb{E} X^{p-1}, \quad p>0 .
\end{aligned}
$$

We will verify that, for $p>0$, provided $\mathbb{E}\left[(1-A)^{-1}\right]<\infty$, (3.7) coincides with the following limit:

$$
\begin{aligned}
\mathbb{E} X^{p} & =\Gamma(p+1) \lim _{n \rightarrow \infty} \prod_{k=1}^{n} \frac{g(p+k)}{g(k)} \\
& =\Gamma(p+1) \prod_{k=1}^{\infty} \frac{\phi(p+k)}{\phi(k)} \frac{1-(1-a) \mathbb{E} A^{k-1}}{1-(1-a) \mathbb{E} A^{p+k-1}} .
\end{aligned}
$$

For the convergence of $\prod_{k=1}^{\infty} g(p+k), p \geq 0$, to a finite positive limit, we verify that

$$
\sum_{k=1}^{\infty}|g(p+k)-1|<\infty
$$

However, for some constant $c>0$,

$$
\begin{aligned}
\sum_{k=1}^{\infty}|g(p+k)-1| & =\sum_{k=1}^{\infty} \frac{|-f(p+k)+(1-a) f(p+k-1)|}{1-(1-a) f(p+k-1)} \\
& \leq c \sum_{k=0}^{\infty} f(p+k) \\
& \leq c \mathbb{E}\left[(1-A)^{-1}\right] \\
& <\infty .
\end{aligned}
$$

Therefore, (3.11) is satisfied and the infinite products in (3.7) have finite positive limits. Now we proceed as in the proof of Proposition 7 of [23]. Consider the function

$$
\psi(p)=\mathbb{E}\left(X^{p-1}\right) \prod_{k=1}^{\infty} \frac{g(k)}{g(p+k-1)} .
$$


In view of (3.10) it satisfies the relations $\psi(p+1)=p \psi(p)$ for $p>0$ and also $\psi(1)=1$. We will show that $\psi$ is the gamma function. As in [23], we will use the Bohr-Mollerup theorem (see [2]), according to which it remains to verify that $\log \psi$ is convex on $(0, \infty)$. We have

$$
\log \psi(p)=\log \left(\prod_{k=1}^{\infty} \frac{g(k)}{g(p+k-1)}\right)+\log \mathbb{E} X^{p-1} .
$$

Following [23], the second derivative of $\log \mathbb{E} X^{p-1}$ is nonnegative. Direct calculation shows that the second derivative of $\sum_{k=1}^{\infty} \log (g(k) / g(p+k-1))$ is nonnegative as well.

Now, in order to indicate that $\left(B_{t}\right)$ and $\left(X_{t}\right)$ depend on $a$, we write $\left(B_{t}^{(a)}\right)$ and $\left(X_{t}^{(a)}\right)$. Of course, $B^{(a)} \stackrel{\mathrm{D}}{\rightarrow} B^{(1)}$ as $a \uparrow 1$, and the limiting random variable has a standard exponential distribution. In our context, it is natural to ask whether the moments $\mathbb{E}\left(X^{(a)}\right)^{p}$ converge to $\mathbb{E}\left(X^{(1)}\right)^{p}$ as $a \uparrow 1$. The answer is indeed positive as follows from the discussion in Example 2.2. Under the assumptions of Lemma 3.3, we have, for $p>0$, as $a \uparrow 1$,

$$
X^{(a)} \stackrel{\mathrm{D}}{\rightarrow} X^{(1)} \quad \text { and } \quad \mathbb{E}\left(X^{(a)}\right)^{p} \rightarrow \mathbb{E}\left(X^{(1)}\right)^{p} .
$$

3.1.4. Geometric $\alpha$-stable $B$. In the previous examples we considered light-tailed distributions of $B$. The present example shows that $B$ can have a very heavy-tailed distribution, while the exponential benchmark (Example 2.1) is still useful for determining the moments of $X$.

We assume that

$$
B_{n}=E_{n}^{1 / \alpha} C_{n}, \quad n \in \mathbb{Z},
$$

where $\left(E_{n}\right)$ is an i.i.d. standard exponential sequence, $\left(C_{n}\right)$ is an i.i.d. sequence of strictly $\alpha$-stable random variables for some $0<\alpha \leq 2$ (cf. [32]), $A>0$ a.s., and $\left(A_{n}\right),\left(E_{n}\right)$, and $\left(C_{n}\right)$ are independent. Then (3.12) defines a strictly geometric $\alpha$-stable random variable; see [26] and [27].

Let $Z$ be an $\alpha$-stable Lévy motion on $[0, \infty)$ such that $Z_{1} \stackrel{\mathrm{D}}{=} C_{1}$ and $N_{t}=\#\left\{i \geq 1: \Gamma_{n} \leq t\right\}$, $t \geq 0, \Gamma_{0}=0, \Gamma_{n}=E_{1}+\cdots+E_{n}, n \geq 1$, be the Poisson process generated by $\left(E_{n}\right)$, $\xi_{t}=\sum_{i=1}^{N_{t}} \log A_{i}, t \geq 0$. Also, assume that $\bar{Z},\left(E_{n}\right)$, and $\left(A_{n}\right)$ are independent. The stationary solution to $X_{n}=A_{n} X_{n-1}+B_{n}, n \in \mathbb{Z}$, has the representation in law

$$
X \stackrel{\mathrm{D}}{=} \int_{0}^{\infty} \mathrm{e}^{\xi_{t}} \mathrm{~d} Z_{t}
$$

Indeed, recalling that $Z_{E_{1}} \stackrel{\mathrm{D}}{=} E_{1}^{1 / \alpha} C_{1}$, we have

$$
\begin{aligned}
\int_{0}^{\infty} \mathrm{e}^{\xi_{t}} \mathrm{~d} Z_{t} & =\sum_{k=1}^{\infty} \exp \left(\sum_{t=1}^{k} \log A_{i}\right)\left(Z_{\Gamma_{k}}-Z_{\Gamma_{k-1}}\right) \\
& \stackrel{\mathrm{D}}{=} \sum_{k=1}^{\infty} A_{1} \cdots A_{k} E_{k}^{1 / \alpha} C_{k} .
\end{aligned}
$$

Write $Y_{0}=\int_{0}^{\infty} \mathrm{e}^{\alpha \xi_{t}} \mathrm{~d} t$, and assume that $Y_{0}$ and $C_{1}$ are independent. By the strict $\alpha$-stability of $Z$,

$$
X \stackrel{\mathrm{D}}{=} C_{1} Y_{0}^{1 / \alpha}
$$


and then, for $p<\alpha$,

$$
\mathbb{E}|X|^{p}=\mathbb{E}|C|^{p} \mathbb{E} Y_{0}^{p / \alpha},
$$

where $\mathbb{E}|C|^{p}$ is a known constant (see [34, Chapter 2]). It is not difficult to see that

$$
Y_{0} \stackrel{\mathrm{D}}{=} A_{1}^{\alpha} Y_{0}+E_{1}
$$

where $A_{1}, E_{1}$, and $Y_{0}$ are independent. Now, the moments of $Y_{0}$ can be determined by using (2.5) and, in a similar way, one can determine the moments of the positive and negative parts of $X$.

\subsection{The case when $B$ is bounded}

In Section 1 we mentioned a popular class of models from financial time series analysis, the ARCH-GARCH family, and the fact that it is common to analyze the fractional moments of such processes. The $\operatorname{GARCH}(1,1)$ squared volatility process $\left(\sigma_{t}^{2}\right)$ is described by the stochastic recurrence equation (1.6). In this case, $B=\alpha_{0}$ is a constant.

The goal of this section is to study the fractional moments of $X$ for bounded $B$. Using the same idea as above, we can obtain similar formulae for recursion (1.1) with bounded $A$ and $B$.

Proposition 3.1. Assume that $A$ and $B$ are independent, and that $0 \leq A \leq a<1,0 \leq B \leq b$ a.s. for some positive constants $a, b$. Write $f(p)=\mathbb{E} A^{p}$ and $g(p)=\mathbb{E} B^{p}, p>0$. Then the following relation holds for $p>0$ :

$$
\begin{aligned}
\mathbb{E} X^{p}= & \sum_{n=0}^{\infty} \frac{p(p-1) \cdots(p-n+1)}{n !} \frac{f(n) g(p-n)}{\phi(n)} \\
& \times \sum_{k_{1}=0}^{n-1} \frac{f\left(k_{1}\right) g\left(n-k_{1}\right)}{\phi\left(k_{1}\right)} \sum_{k_{2}=0}^{k_{1}-1} \frac{f\left(k_{2}\right) g\left(k_{1}-k_{2}\right)}{\phi\left(k_{2}\right)} \cdots \sum_{k_{n}=0}^{k_{n-1}-1} \frac{f\left(k_{n}\right) g\left(k_{n-1}-k_{n}\right)}{\phi\left(k_{n}\right)} \\
& \times\left(\begin{array}{c}
k_{1}-k_{2} k_{2}-k_{3} \cdots k_{n-1}-k_{n} k_{n} \\
k_{1}
\end{array}\right) .
\end{aligned}
$$

Proof. We start by observing that $A_{1} X_{0} \leq a b(1-a)^{-1}$. Thus, if $a<(b+1)^{-1}$, a Taylor expansion of $\left(B_{1}+x\right)^{p}$ for $0 \leq x \leq a b(1-a)^{-1}<1$ yields

$$
\mathbb{E}\left(B_{1}+A_{1} X_{0}\right)^{p}=\sum_{n=0}^{\infty} \frac{p(p-1) \cdots(p-n+1)}{n !} f(n) \mathbb{E} B^{p-n} \mathbb{E} X^{n} .
$$

Then multiple use of (1.5) yields (3.13).

For any $c>0$, write $B_{t}(c)=B_{t} / c$ and $X_{t}(c)=X_{t} / c$. The stochastic recurrence equation (1.1) is equivalent to

$$
X_{t}(c)=A_{t} X_{t-1}(c)+B_{t}(c), \quad t \in \mathbb{Z},
$$

and $\mathbb{E} X^{p}=c^{p} \mathbb{E}(X(c))^{p}$. Since $B(c) \leq b / c$ a.s. and $X(c) \leq a(b / c)(1-a)^{-1}$, the above calculations are valid for the new equation (3.14) under the condition $a<(b / c+1)^{-1}$. Thus, if $c$ is sufficiently large, the value $a$ may be arbitrarily close to 1 and all moment calculations can be extended for arbitrary $a<1$ and $b>0$.

Remark 3.1. The condition $a<1$ is crucial for the above calculations. If $\mathbb{P}(A=1)>0$ then all of the moments $\mathbb{E} X^{p}, p>0$, can still be finite. For example, assume that we have 
$q=\mathbb{P}(A=0)=1-\mathbb{P}(A=1) \in(0,1)$ and $B \equiv 1$ a.s. Then $X$ has a geometric distribution with parameter $q$ and the moments are given by

$$
\mathbb{E} X^{p}=(1-q) \sum_{n=1}^{\infty} q^{n-1} n^{p}, \quad p>0 .
$$

The latter example extends to the case when $q=\mathbb{P}(A=0)=1-\mathbb{P}(A=a) \in(0,1)$ for some $a>0$. Then $X=\sum_{n=1}^{N} a^{n}$, where $N$ is geometric with success probability $q$ and the moments are given by

$$
\mathbb{E} X^{p}=(1-q) \sum_{n=1}^{\infty} q^{n-1}\left(\sum_{k=1}^{n} a^{k}\right)^{p} .
$$

For $a \neq 1$, this turns into

$$
\mathbb{E} X^{p}=(1-q) \frac{a^{p}}{|1-a|^{p}} \sum_{n=1}^{\infty} q^{n-1}\left|1-a^{n}\right|^{p},
$$

which is finite for $q a^{p}<1$.

In the remainder of this section, we assume that $B \equiv 1$ a.s. This case can be understood as a limiting case when $B$ is Erlang distributed and the parameter of the Erlang distribution tends to $\infty$. Indeed, consider the sequence of stochastic recurrence equations $X_{t}^{(n)}=A_{t} X_{t-1}^{(n)}+B_{t}^{(n)}$, $t \in \mathbb{Z}$, where $\left(B_{t}^{(n)}\right)$ is an i.i.d. sequence with $B^{(n)} \stackrel{\mathrm{D}}{=} n^{-1}\left(E_{1}+\cdots+E_{n}\right)$ for an i.i.d. sequence $\left(E_{t}\right)$ with standard exponential marginal distribution. We also assume that $\left(A_{t}\right)$ and $\left(B_{t}^{(n)}\right)$ are independent. By the strong law of large numbers, $B_{t}^{(n)} \rightarrow 1$ a.s. as $n \rightarrow \infty$ for every $t$ and we also have $\mathbb{E}\left|B^{(n)}-1\right|^{p} \rightarrow 0$ for every $p>0$. Lemma 2.2 now yields $\mathbb{E}\left|X^{(n)}\right|^{p} \rightarrow \mathbb{E}|X|^{p}$.

A change of the indices in (3.13) and $g \equiv 1$ yield the alternative expression

$$
\begin{aligned}
\mathbb{E} X^{p}=\sum_{j=1}^{\infty} \sum_{n_{1}=1}^{\infty} \cdots \sum_{n_{j}=1}^{\infty} & \frac{p(p-1) \cdots\left(p-\left(n_{1}+\cdots+n_{j}\right)+1\right)}{\prod_{d=1}^{j} n_{d} !} \\
& \times \frac{f\left(n_{1}\right)}{\phi\left(n_{1}\right)} \frac{f\left(n_{1}+n_{2}\right)}{\phi\left(n_{1}+n_{2}\right)} \cdots \frac{f\left(n_{1}+\cdots+n_{j}\right)}{\phi\left(n_{1}+\cdots+n_{j}\right)} .
\end{aligned}
$$

This formula can be easily evaluated and converges very fast. We illustrate this aspect for $A$ with a uniform distribution on $(0, a), a<1$. Then

$$
\begin{aligned}
\sum_{j=1}^{\infty} \sum_{n_{1}=1}^{\infty} \cdots \sum_{n_{j}=1}^{\infty} & \frac{p(p-1) \cdots\left(p-\left(n_{1}+\cdots+n_{j}\right)+1\right)}{\prod_{d=1}^{j} n_{d} !} \frac{a^{n_{1}}}{\left(n_{1}+1\right)-a^{n_{1}}} \\
& \times \frac{a^{n_{1}+n_{2}}}{\left(n_{1}+n_{2}+1\right)-a^{n_{1}+n_{2}}} \cdots \frac{a^{n_{1}+\cdots+n_{j}}}{\left(n_{1}+\cdots+n_{j}+1\right)-a^{n_{1}+\cdots+n_{j}}} .
\end{aligned}
$$

In Figure 1 we approximate the latter expression for $p=0.6$ and values $a \in(0,1)$ by replacing all infinite sums in (3.15) by the sums truncated at some integer $M \geq 1$. The value $M$ is indicated on the $x$-axis. The corresponding absolute change of the approximated moment from $M$ to $M+1$ is tabulated in Table 1 .

We mention for the sake of completeness that the calculation of Goldie's constant $c_{+}$in (1.4) in the case $B \equiv 1$ a.s. has been addressed in [19]. However, since we assume that $A<1$ a.s., the results of this section are beyond the Kesten-Goldie setting. 


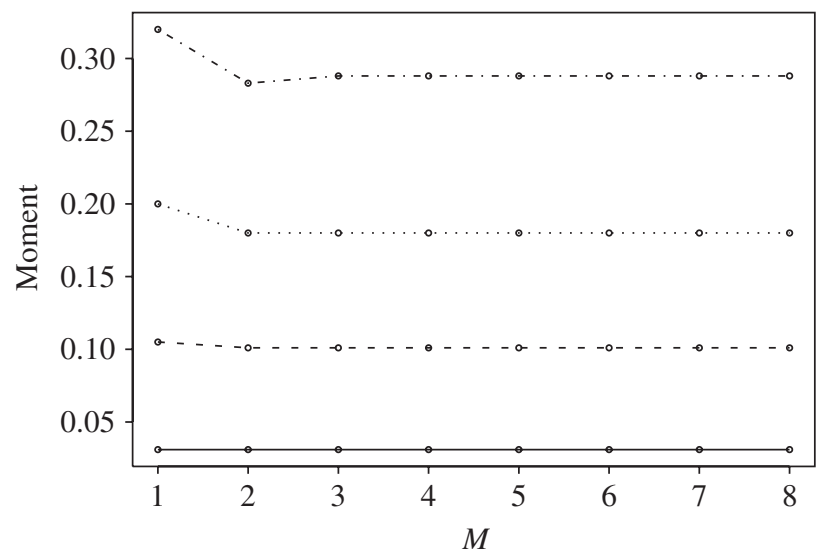

FIGURE 1: Approximation of $\mathbb{E} X^{0.6}$ for $B=1$ and $A$ uniform on $(0, a)$, taking into account only the first $M$ (indicated on the $x$-axis) summands in each of the infinite series in (3.15). The curves from bottom to top correspond to $a=0.1,0.3,0.5,0.7$.

TABLE 1: Absolute values of the differences of the approximated moments for $p=0.6$. The symbol $\Delta_{M, M+1}$ describes the absolute value of the change of the approximation from $M$ to $M+1$.

\begin{tabular}{cccccccc}
\hline$a$ & $\Delta_{12}$ & $\Delta_{23}$ & $\Delta_{34}$ & $\Delta_{45}$ & $\Delta_{56}$ & $\Delta_{67}$ & $\Delta_{78}$ \\
\hline 0.1 & $4 \times 10^{-4}$ & $1 \times 10^{-5}$ & $6 \times 10^{-7}$ & $4 \times 10^{-8}$ & $2 \times 10^{-9}$ & $1 \times 10^{-10}$ & $1 \times 10^{-11}$ \\
0.3 & $5 \times 10^{-3}$ & $3 \times 10^{-4}$ & $5 \times 10^{-5}$ & $8 \times 10^{-6}$ & $1 \times 10^{-6}$ & $3 \times 10^{-7}$ & $6 \times 10^{-8}$ \\
0.5 & $2 \times 10^{-2}$ & $2 \times 10^{-3}$ & $3 \times 10^{-4}$ & $7 \times 10^{-5}$ & $2 \times 10^{-5}$ & $6 \times 10^{-6}$ & $2 \times 10^{-6}$ \\
0.7 & $4 \times 10^{-2}$ & $4 \times 10^{-3}$ & $9 \times 10^{-4}$ & $3 \times 10^{-4}$ & $9 \times 10^{-5}$ & $4 \times 10^{-5}$ & $3 \times 10^{-5}$ \\
\hline
\end{tabular}

\section{Acknowledgement}

We would like to thank the referee for a careful reading of our paper. The referee's remark led us to restructure the content of the paper, making it more accessible.

\section{References}

[1] Alsmeyer, G., Iksanov, A. And Rösler, U. (2009). On distributional properties of perpetuities. J. Theoret. Prob. 22, 666-682.

[2] Andrews, G. E., Askey, R. And Roy, R. (1999). Special Functions. Cambridge University Press.

[3] Basrak, B., Davis, R. A. AND Mikosch, T. (2002). A characterization of multivariate regular variation. Ann. Appl. Prob. 12, 908-920.

[4] Basrak, B., Davis, R. A. ANd Mikosch, T. (2002). Regular variation of GARCH processes. Stoch. Process. Appl. 99, 95-115.

[5] Behme, A., Lindner, A. And Maller, R. (2011). Stationary solutions of the stochastic differential equation $d V_{t}=V_{t}-d U_{t}+d L_{t}$ with Lévy noise. Stoch. Process. Appl. 121, 91-108.

[6] Bollerslev, T. (1986). Generalized autoregressive conditional heteroskedasticity. J. Econometrics 31, 307-327.

[7] Boxma, O., Kella, O. And Perry, D. (2011). On some tractable growth-collapse processes with renewal collapse epochs. In New Frontiers in Applied Probability (J. Appl. Prob. Spec. Vol. 48A), eds P. Glynn, T. Mikosch and T. Rolski, Applied Probability Trust, Sheffield, pp. 217-234.

[8] Breiman, L. (1965). On some limit theorems similar to the arc-sin law. Theory Prob. Appl. 10, 323-331.

[9] Brockwell, P. J. ANd Lindner, A. (2009). Existence and uniqueness of stationary Lévy-driven CARMA processes. Stoch. Process. Appl. 119, 2660-2681. 
[10] Carmona, P., Petit, F. and Yor, M. (1997). On the distribution and asymptotic results for exponential functionals of Lévy processes. In Exponential Functionals and Principle Values Related to Brownian motion, ed. M. Yor, Revista Matematica Iberoamericana, Madrid, pp. 73-130.

[11] Collamore, J. F. (2009). Random recurrence equations and ruin in a Markov-dependent stochastic economic environment. Ann. Appl. Prob. 19, 1404-1458.

[12] Diaconis, P. And Freedman, D. (1999). Iterated random functions. SIAM Rev. 41, 45-76.

[13] Dufresne, D. (1990). The distribution of a perpetuity, with applications to risk theory and pension funding. Scand. Actuarial J. 1990, 39-79.

[14] Dufresne, D. (1996). On the stochastic equation $\mathcal{L}(X)=\mathscr{L}[B(X+C)]$ and a property of gamma distributions. Bernoulli 2, 287-291.

[15] Dufresne, D. (1998). Algebraic properties of beta and gamma distributions, and applications. Adv. Appl. Math. 20, 285-299.

[16] Dufresne, D. (2010). G distributions and the beta-gamma algebra. Electron. J. Prob. 15, 2163-2199.

[17] Dumas, V., Guillemin, F. And Robert, P. (2002). A Markovian analysis of additive-increase multiplicativedecrease algorithms. Adv. Appl. Prob. 34, 85-111.

[18] ENGLE, R. F. (1982). Autoregressive conditional heteroscedasticity with estimates of the variance of United Kingdom inflation. Econometrica 50, 987-1007.

[19] Enriquez, N., SABot, C. And Zindy, O. (2009). A probabilistic representation of constants in Kesten's renewal theorem. Prob. Theory Relat. Fields 144, 581-613.

[20] Gjessing, H. K. and Paulsen, J. (1997). Present value distributions with applications to ruin theory and stochastic equations. Stoch. Process. Appl. 71, 123-144.

[21] Goldie, C. M. (1991). Implicit renewal theory and tails of solutions of random equations. Ann. Appl. Prob. 1, 126-166.

[22] Goldie, C. M. And Grübel, R. (1996). Perpetuities with thin tails. Adv. Appl. Prob. 28, 463-480.

[23] Guillemin, F., Robert, P. and Zwart, B. (2004). AIMD algorithms and exponential functionals. Ann. Appl. Prob. 14, 90-117.

[24] Hirsch, F. AND Yor, M. (2013). On the Mellin transforms of the perpetuity and the remainder variables associated to a subordinator. Bernoulli 19, 1350-1377.

[25] Kesten, H. (1973). Random difference equations and renewal theory for products of random matrices. Acta Math. 131, 207-248.

[26] Klebanov, L. B., Maniya, G. M. and Melamed, I. A. (1985). A problem of Zolotarev and analogs of infinitely divisible and stable distributions in a scheme for summing a random number of random variables. Theory Prob. Appl. 29, 791-794.

[27] Kozubowski, T. J. (2000). Exponential mixture representation of geometric stable distributions. Ann. Inst. Statist. Math. 52, 231-238.

[28] Löpker, A. H. And van LeeuwaArden, J. S. H. (2008). Transient moments of the TCP window size process. J. Appl. Prob. 45, 163-175.

[29] Maulik, K. AND Zwart, B. (2006). Tail asymptotics for exponential functionals of Lévy processes. Stoch. Process. Appl. 116, 156-177.

[30] Pitman, J. And Yor, M. (2003). Infinitely divisible laws associated with hyperbolic functions. Canad. J. Math. 55, 292-330.

[31] Rachev, S. T. (1991). Probability Metrics and the Stability of Stochastic Models. John Wiley, Chichester.

[32] Samorodnitsky, G. And TaqQu, M. (1994). Stable Non-Gaussian Random Processes. Chapman \& Hall, New York.

[33] VervaAt, W. (1979). On a stochastic difference equation and a representation of nonnegative infinitely divisible random variables. Adv. Appl. Prob. 11, 750-783.

[34] Zolotarev, V. M. (1986). One-Dimensional Stable Distributions (Trans. Math. Monogr. 65). American Mathematical Society, Providence, RI. 